\title{
Diminuição no uso de bebidas alcoólicas e a violência pelo parceiro íntimo
}

\author{
Decreased use of alcoholic beverages and intimate partner violence \\ Disminución del uso de bebidas alcohólicas y violencia de pareja
}

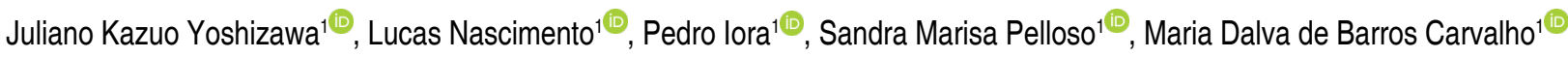 \\ ${ }^{1}$ Universidade Estadual de Maringá, Maringá, Paraná, Brasil.
}

\section{Resumo}

Introdução: A violência praticada por parceiro íntimo refere-se ao comportamento de parceiros ou exparceiros íntimos que resulta em dano físico, sexual ou psicológico, incluindo agressão física, coerção sexual, abuso psicológico e comportamento controlador. Sabe-se que o etilismo está associado ao aumento de tal violência. Objetivo: Analisar se a acompanhante do paciente em abstinência alcoólica referia menor índice de violência nesse período em relação ao tempo em que o mesmo fazia abuso de álcool. Métodos: Estudo observacional transversal no qual foram selecionados homens ex-etilistas atendidos no CAPSad de Maringá e suas parceiras. Foi utilizado um questionário para violência contra parceiro (HITS) composto de 4 perguntas objetivas, cuja pontuação varia de 4 até 20 . Valores iguais ou superiores a 10 indicam violência. Resultados: Foram entrevistadas 53 mulheres de diversas faixas etárias e escolaridades. Desse total, $84,9 \%$ das participantes apresentaram pontuações menores no teste com o parceiro em abstinência em relação ao período em que o mesmo estava em uso/ abuso de álcool. Das mulheres $15,1 \%$ não notaram diferença no nível de violência do acompanhante, estivesse ele em uso ou em abstinência alcoólica. Conclusões: Demonstrou-se claramente que o fato de cessar o consumo de bebidas alcoólicas reduziu o índice de violência infligida pelo parceiro.

Palavras-chave: Alcoolismo; Abstinência de Álcool; Violência por Parceiro Íntimo.

Como citar: Yoshizawa JK, Carvalho MDB, Nascimento L, lora P, Pelloso SM. Diminuição no uso de bebidas alcoólicas e a violência pelo parceiro íntimo. Rev Bras Med Fam Comunidade. 2020;15(42):2263. https://doi.org/10.5712/rbmfc15(42)2263
Autor correspondente: Juliano Kazuo Yoshizawa. E-mail: julianomfc@gmail.com Fonte de financiamento: declaram não haver.

Parecer CEP:

Aprovado. Número do parecer: 2.230.642. Procedência e revisão por pares: peer reviewed.

Recebido em: 25/10/2019. Aprovado em: 16/12/2019. 


\begin{abstract}
Introduction: Intimate partner violence refers to the behavior of intimate partners or ex-partners resulting in physical, sexual, or psychological harm, including physical aggression, sexual coercion, psychological abuse, and controlling behavior. It is known that alcoholism is associated with increase of this type of violence. Objective: The aim of this study was to analyze whether the partner of the patient in alcohol withdrawal reported a lower rate of violence in this period compared with the period of alcohol abuse. Methods: cross-sectional study in which we selected male former drinkers attended at the CAPSad of Maringá, and their partners. A questionnaire regarding partner violence (HITS), consisting of 4 objective questions with scores ranging from 4 to 20, was used. Values equal to or greater than 10 indicate violence. Results: We interviewed 53 women of different ages and schooling. Of this total, $84.9 \%$ of the participants had scores in the test with the partner in withdrawal lower than in the period in which he was in use/abuse of alcohol. $15.1 \%$ of the women did not notice a difference in the level of violence of their partners, whether he was in use or withdrawal. Conclusions: It was clearly demonstrated that the cessation of alcohol consumption reduced the rate of violence inflicted by the partner.
\end{abstract}

Keywords: Alcoholism; Alcohol Abstinence; Intimate Partner Violence.

\title{
Resumen
}

Introducción: La violencia practicada por un compañero íntimo se refiere al comportamiento de parejas o ex compañeros íntimos que resultan en daño físico, sexual o psicológico, incluyendo agresión física, coerción sexual, abuso psicológico y comportamiento controlador. Se sabe que el etilismo está asociado al aumento de tal violencia. Objetivo: analizar si la compañera del paciente en abstinencia alcohólica refería menor índice de violencia en ese período en relación al tiempo en que el mismo hacía abuso de alcohol. Método: estudio tranversal en el que se seleccionaron hombres ex etilistas del CAPSad de Maringá y sus parejas. Se utilizó un cuestionario para violencia contra parejas (HITS) compuesto de 4 preguntas objetivas cuya puntuación varía de 4 a 20. Valores igual ou superiores a 10 indican violencia. Resultados: Se entrevistaron a 53 mujeres de diversas edades y escolaridades. De ese total, el $84,9 \%$ de las participantes presentó puntuaciones menores en la prueba con el compañero en abstinencia en relación al período en que el mismo estaba en uso / abuso de alcohol. El 15,1\% de las mujeres no notaron diferencia en el nivel de violencia del acompañante, esté en uso o abstinencia alcohólica. Conclusión: Se demostró claramente que el hecho de cesar el consumo de bebidas alcohólicas redujo el índice de violencia infligido por el compañero.

Palabras clave: Alcoholismo; Abstinencia de Alcohol; Violencia de Pareja.

\section{Introdução}

Para entender a complexidade da violência contra a mulher é necessário refletir e compreender as questões de gênero. Trata-se de uma construção histórica e sociocultural, que aborda as diferenças do comportamento feminino e do masculino. Apesar das conquistas sociais alcançadas pela mulher ao longo dos anos, elas ainda são descritas em discursos machistas que as consideram passivas, frágeis, com emoções à flor da pele e submissas. Já os homens são vistos como fortes, racionais e com poder de domínio. ${ }^{1}$

Segundo a Organização Mundial de Saúde (OMS), mais de um terço de todas as mulheres do mundo são vítimas de violência física sendo perpetrado, na maioria das vezes, pelo cônjuge ou namorado. ${ }^{2}$

O uso abusivo de álcool e uso ilícito de drogas são fatores de risco frequentemente associados à experiência e à perpetração de violência praticada pelo parceiro íntimo, ${ }^{3,4}$ assim como há evidências de que o consumo de álcool por um ou ambos os parceiros está associado ao aumento desse tipo de violência. ${ }^{5}$

De acordo com pesquisa realizada no Brasil sobre a violência contra as mulheres e relações de gênero no espaço público e privado, estimou-se que entre as 2.365 mulheres de 25 Estados da Federação, 34\% estavam sujeitas a violência no espaço doméstico.

A cada 2 minutos, cinco mulheres são agredidas violentamente. Os atos violentos resultam na perda de um ano de vida saudável a cada 5 anos de submissão às agressões. ${ }^{1}$ 
Esses números crescentes e alarmantes da violência contra a mulher podem estar associados a uma série de fatores como: a baixa escolaridade, que gera uma dependência financeira por parte da mulher, a dependência afetiva, a relação de poder exercida pelo homem, e o uso de álcool pelo companheiro. ${ }^{2}$ Além disso, há evidências que relacionam experiências adversas na infância, impulsividade, depressão e ansiedade com o consumo de álcool e a violência por parceiro íntimo. ${ }^{6,7}$

Uma pesquisa realizada em âmbito nacional sobre o padrão de consumo de álcool pela população brasileira associou o uso de álcool a algum tipo de violência praticada contra a mulher. Segundo as entrevistadas (companheiras dos usuários), 25\% dos parceiros se irritaram após ingestão do álcool, 12\% disseram ter iniciado uma discussão devido ao uso de álcool pelo parceiro, o que levou à constatação de que o álcool acarreta uma mudança de humor e favorece a agressão. ${ }^{8}$

Este estudo é um dos poucos que procura avaliar a violência pelo parceiro íntimo em abstinência e não no período em que o mesmo fazia abuso de álcool e tem como objetivo verificar a percepção das parceiras quanto a comportamentos de violência.

\section{Material e Métodos}

Estudo observacional transversal retrospectivo, realizado em um município do sul do Brasil no período de outubro a dezembro de 2017.

Os sujeitos dessa pesquisa foram parceiras de indivíduos do sexo masculino com problemas relacionados ao álcool que frequentam o CAPSad (Centro de Atenção Psicossocial Álcool e Drogas) da cidade de Maringá, estado do Paraná. Os indivíduos selecionados estavam obrigatoriamente em abstinência alcoólica. Foram consideradas apenas as pessoas que acompanhavam os pacientes ao longo do período em que fizeram abuso de álcool e mantiveram-se juntas até a abstemia, independente de estarem casados, namorando ou em união estável. A abordagem foi realizada somente para os casais que procuravam pelo CAPSad, ou seja, não foi realizado contato com parceiros que não estavam presentes no momento do atendimento. Foram excluídos aqueles que faziam uso de cigarro e qualquer tipo de substância ilícita, além dos portadores de transtornos psiquiátricos graves, ou seja, aqueles que não conseguiram ser manejados em UBS.

O número de parceiras $(n)$ necessário para compor a amostra, com o intuito de estimar o escore do instrumento HITS, foi calculado de acordo com a fórmula9:

$$
n=\left(\frac{\mathrm{Z} \alpha / 2 \cdot \delta}{E}\right)^{2}
$$

em que $Z_{\alpha / 2}$ é o valor crítico para o nível de confiança, fixado em $95 \%$, $\delta$ é o desvio padrão da variável de interesse, para o qual considerou-se a estimativa de 2,75 , obtida no estudo realizado no qual e $E$ é o erro amostral, que expressa o erro máximo admitido entre a estimativa e o valor real do parâmetro fixado em 0,75 pontos $^{10}$. Dessa forma, o tamanho amostral, obtido com o auxílio do ambiente estatístico $R$ ( $R$ Core Team), versão 3.3.1, foi de $n=52$ mulheres para estimar o escore do HITS.

As parceiras dos mesmos responderam um questionário para verificar a presença de violência durante o período em que os pacientes faziam uso abusivo do álcool e no período de abstinência. Foram 
divididas quanto à escolaridade em: ensino fundamental, ensino médio e ensino superior. Já em relação à idade em anos foram divididas nos seguintes grupos: 18-29, 30-39, 40-49, 50-59 e >60.

As entrevistas foram conduzidas pelos próprios autores, que eram professor e alunos (todos do sexo masculino) do curso de graduação em medicina da Universidade Estadual de Maringá, que não tinham nenhum papel na assistência à saúde dos pacientes. O professor tinha tais qualificações e treinou pessoalmente os estudantes para a condução das entrevistas, no próprio CAPSad (local com acesso livre para qualquer paciente etilista) preferencialmente em grupos onde participam algum profissional da saúde, pacientes em tratamento e suas acompanhantes. Os eleitos para a pesquisa foram todos os pacientes e as acompanhantes dos mesmos que aceitaram participar da mesma, que estavam em acompanhamento no CAPSad, incluindo também as pessoas que já participavam dos grupos de acolhimento. Todos foram informados previamente sobre o projeto e preencheram o Termo de Consentimento Livre e Esclarecido. Em seguida foi distribuído um impresso contendo o questionário HITS (Hurt,Insult, Threaten,Scream), que foi escolhido por ser facilmente aplicável dentre os vários modelos possíveis para rastreamento de violência causada pelo parceiro. Tal instrumento possui sensibilidade de $75 \%$ e especificidade $83 \%{ }^{11}$ aproximadamente. É validado internacionalmente ${ }^{12,13}$ e também no Brasil ${ }^{14}$. O teste é composto por 4 perguntas, listadas a seguir:

1-Com que frequência o seu parceiro machuca você fisicamente?

2-Com que frequência o seu parceiro insulta você ou não deixa você falar?

3-Com que frequência o seu parceiro ameaça você com injúria?

4-Com que frequência o seu parceiro grita ou amaldiçoa você?

São possíveis 5 respostas para cada pergunta: nunca (1 ponto), raramente (2 pontos), às vezes (3 pontos), relativamente frequente (4 pontos) e frequentemente (5 pontos).Não há uma definição objetiva do que seria considerado "às vezes" ou "frequentemente", por exemplo, ficando a resposta a critério do entendimento do entrevistado. Cada questão pode gerar de 1 até 5 pontos e uma somatória igual ou superior a 10 pontos é considerada positiva para violência doméstica.

Aplicou-se o questionário apenas para as parceiras dos pacientes etilistas, considerando dois momentos distintos, um no qual o mesmo ainda fazia abuso de álcool e outro em que ele já estava em abstinência.

Foram realizadas ainda entrevistas livres onde foi perguntado qual era a diferença entre o comportamento dos companheiros em casa, no trabalho e com os amigos no período em que usavam bebidas alcoólicas e em abstinência. Foram entrevistadas todas aquelas que aceitaram discorrer sobre o caso, além de preencher o questionário. As conversas foram registradas via gravador em aparelho celular e analisadas individualmente por um dos autores (JKY).

Os dados obtidos foram digitados em planilha do programa Microsoft Excel 2013 e analisados estatisticamente com o auxílio do Software Statistica Single Userversão 13.2 e SAS (Statistical Analysis System) versão 9.3. Para a análise foram utilizados testes paramétricos, pois os autores assumiram normalidade dos dados já que se trata de variáveis dispostas em escala de Likert. Após verificação de normalidade por meio do teste Shapiro wilk, optou-se pela estatística paramétrica. As variáveis qualitativas foram dispostas em tabelas simples com apresentação de percentuais. Já as variáveis quantitativas foram dispostas em uma tabela com apresentação de média, desvio padrão e intervalo de confiança com nível de 
confiança de $95 \%$ e teste $t$ de Student para comparar os questionamentos quando o companheiro estava ou não fazendo uso de bebidas alcoólicas. E por fim, foi realizada uma análise de medidas repetidas para se verificar a interação das variáveis observadas no questionário HITS, idade e escolaridade em relação ao uso ou abstinência.

O estudo foi aprovado pelo Comitê de Ética da Universidade Estadual de Maringá sob o parecer número 2.230.642 do ano de 2017.

Serão compartilhados dados referentes à idade e escolaridade das entrevistadas por tempo indeterminado, desde a publicação do presente trabalho via impressa e digital, disponíveis em https://doi. org/10.5281/zenodo.3576658.

Nenhum paciente esteve envolvido no planejamento da pesquisa.

\section{Resultados}

Levando em consideração os critérios de inclusão e exclusão no período de outubro até dezembro de 2017, obteve-se um total de 53 mulheres, as quais foram entrevistadas. Não houve nenhum entrevistado do sexo masculino, pois não encontramos nenhum paciente que tivesse relação homoafetiva. Foram abordadas todas as parceiras que acompanhavam os pacientes eleitos para o estudo. Não foram contabilizadas quantas se negaram a participar da entrevista e nem investigado o motivo da recusa.

A idade média ( \pm desvio-padrão) das entrevistadas foi de $36,5 \pm 12,8$ anos. A maioria, $66,0 \%(n=35)$ das mulheres estava na faixa etária dos 18 e 39 anos, 28,3\% $(n=15)$ tinham entre 30 e 39 anos, 18,9\% $(n=10)$ com idade de 40 a 49 anos, 15,1\% $(n=8)$ tinham idade acima de 50 anos sendo que $7,5 \%(n=4)$ tinham entre 50 e 59 anos e o mesmo percentual acima de 60 anos.

A maioria das entrevistadas $(58,5 \% ; n=31)$ estudou até o ensino médio (completo ou incompleto), $22,6 \%(n=12)$ cursou o ensino fundamental (completo ou incompleto) e o restante, $18,9 \%(n=10)$ ensino superior (completo ou incompleto) conforme apresentado na Tabela 1.

Tabela 1. Características demográficas das parceiras de pacientes do CAPS-ad que alcançaram abstinência do uso de bebidas alcoólicas. Maringá, Paraná, 2017.

\begin{tabular}{lc}
\hline Idade & \multicolumn{1}{c}{ Média \pm Desvio padrão } \\
& $36,5 \pm 12,8$ \\
\hline Perfil & $\mathrm{n}$ \\
\hline Faixa etária & 20 \\
\hline De 18 a 29 anos & 15 \\
De 30 a 39 anos & 10 \\
De 40 a 49 anos & 4 \\
De 50 a 59 anos & 4 \\
60 anos ou mais & \\
\hline Escolaridade & 12 \\
\hline Ensino fundamental (in)completo & 31 \\
Ensino médio (in)completo & 10 \\
Ensino superior (in)completo & 4 \\
\hline
\end{tabular}


Tanto nas questões do questionário HITS quanto na medida de violência (soma das quatro questões), houve aumento no padrão de violência quando a entrevistada respondeu as questões se referindo ao tempo em que o parceiro fazia uso abusivo de álcool, destacando-se na Tabela 2 que para a medida de violência, esse aumento foi de 4,8 pontos em média, com intervalo de confiança de $95 \%$ de $(3,8 ; 5,8)$. Comparando os resultados com em abstinência e em uso de álcool, a menor diferença observada foi para a primeira questão, relacionada à violência física, para a qual a pontuação média é 0,5 pontos maior no período em que o parceiro fazia uso de álcool, enquanto essa diferença média varia entre 1,3 e 1,5 pontos para as demais questões.

Tabela 2. Pontuação no questionário HITS antes e depois da interrupção do uso de bebidas alcoólicas.

\begin{tabular}{|c|c|c|c|c|c|c|c|c|c|c|c|c|c|c|c|}
\hline \multirow{3}{*}{$\begin{array}{l}\text { Variáveis } \\
\text { Q1 }\end{array}$} & \multicolumn{5}{|c|}{ Em abstinência } & \multicolumn{5}{|c|}{ Uso de álcool } & \multicolumn{5}{|c|}{ Diferença (Uso de álcool - Em abstinência) } \\
\hline & \multirow{2}{*}{$\begin{array}{c}\text { Média } \\
1,1\end{array}$} & \multirow{2}{*}{$\begin{array}{l} \pm \\
\pm\end{array}$} & \multirow{2}{*}{$\begin{array}{c}\begin{array}{c}\text { Desvio } \\
\text { padrão }\end{array} \\
0,4\end{array}$} & \multicolumn{2}{|c|}{$\begin{array}{c}\text { IC } \\
(95 \%)\end{array}$} & \multirow{2}{*}{$\begin{array}{c}\text { Média } \\
1,6\end{array}$} & \multirow{2}{*}{$\begin{array}{l} \pm \\
\pm\end{array}$} & \multirow{2}{*}{$\begin{array}{c}\begin{array}{c}\text { Desvio } \\
\text { padrão }\end{array} \\
0,8\end{array}$} & \multicolumn{2}{|c|}{$\begin{array}{c}\text { IC } \\
(95 \%)\end{array}$} & \multirow{2}{*}{$\begin{array}{c}\text { Média } \\
0,5\end{array}$} & \multirow{2}{*}{$\begin{array}{l} \pm \\
\pm\end{array}$} & \multirow{2}{*}{$\begin{array}{c}\begin{array}{c}\text { Desvio } \\
\text { padrão }\end{array} \\
0,8\end{array}$} & \multicolumn{2}{|c|}{$\begin{array}{c}\text { IC } \\
(95 \%)\end{array}$} \\
\hline & & & & 1,0 & 1,2 & & & & 1,4 & 1,8 & & & & 0,3 & 0,7 \\
\hline Q2 & 1,3 & \pm & 0,6 & 1,1 & 1,4 & 2,8 & \pm & 1,4 & 2,4 & 3,2 & 1,5 & \pm & 1,6 & 1,1 & 2,0 \\
\hline Q3 & 1,2 & \pm & 0,5 & 1,0 & 1,3 & 2,5 & \pm & 1,3 & 2,1 & 2,8 & 1,3 & \pm & 1,4 & 0,9 & 1,7 \\
\hline Q4 & 1,5 & \pm & 0,8 & 1,3 & 1,7 & 3,0 & \pm & 1,4 & 2,6 & 3,4 & 1,5 & \pm & 1,2 & 1,2 & 1,8 \\
\hline Violência & 5,1 & \pm & 1,8 & 4,6 & 5,5 & 9,9 & \pm & 3,7 & 8,8 & 10,9 & 4,8 & \pm & 3,7 & 3,8 & 5,8 \\
\hline
\end{tabular}

IC: Intervalo de Confiança

O padrão de respostas pode ser dividido em 3 grupos:

- $1^{\circ}$ grupo: composto por $15 \%$ das entrevistadas no qual a pontuação foi a mesma com o parceiro em uso e em abstinência (ambas com menos de 10 pontos não caracterizando violência).

- $2^{\circ}$ grupo: composto por $21 \%$ das entrevistadas no qual a pontuação foi maior com o parceiro em uso (porém a pontuação ainda era menor do que 10 pontos tanto no uso como na abstinência), ou seja, não houve violência em ambas as situações.

- $3^{\circ}$ grupo: composto por $64 \%$ das entrevistadas no qual a pontuação foi superior a 10 (caracterizando violência) com o parceiro fazendo abuso de álcool e com pontuação inferior a 10 (sem violência) com o parceiro em abstinência.

Houve ainda uma única entrevistada que relatou que o parceiro ficava mais calmo ao ingerir bebidas alcoólicas.

$\mathrm{Na}$ entrevista livre com as 8 companheiras obteve-se respostas muito semelhantes. A maior parte relatou mudança importante no comportamento do cônjuge em abstinência. Termos como "mudou completamente" foram recorrentes durante as entrevistas.

Comparando a medida de violência total do HITS entre as faixas etárias e graus de escolaridade, tanto antes quanto depois da interrupção do uso de bebidas alcoólicas, observam-se poucas diferenças, como apresentado na Tabela 3. Destaca-se que em relação à faixa etária, no momento de abstinência o menor escore médio foi observado para a faixa etária de 50 anos ou mais e no momento de uso de álcool, a maior pontuação média foi observada para a faixa etária de 18 a 29 anos. Em relação à escolaridade, notase que enquanto a maior pontuação média foi verificada para o grupo com ensino médio (in)completo no momento em abstinência, no momento de uso de álcool esse grupo apresentou a menor média do escore. 
Tabela 3. Pontuação no questionário HITS antes e depois da interrupção do uso de bebidas alcoólicas, de acordo com a faixa etária e escolaridade.

\begin{tabular}{|c|c|c|c|c|c|c|c|c|c|c|}
\hline \multirow{2}{*}{$\begin{array}{l}\text { Variáveis } \\
\text { Faixa etária }\end{array}$} & \multicolumn{5}{|c|}{ Em abstinência } & \multicolumn{5}{|c|}{ Uso de álcool } \\
\hline & Média & \pm & $\begin{array}{l}\text { Desvio } \\
\text { padrão }\end{array}$ & \multicolumn{2}{|c|}{$\begin{array}{c}\text { IC } \\
(95 \%)\end{array}$} & Média & \pm & $\begin{array}{l}\text { Desvio } \\
\text { padrão }\end{array}$ & \multicolumn{2}{|c|}{$\begin{array}{c}\text { IC } \\
(95 \%)\end{array}$} \\
\hline De 18 a 29 anos & 5,2 & \pm & 2,3 & 4,2 & 6,1 & 10,3 & \pm & 3,6 & 8,7 & 11,8 \\
\hline De 30 a 49 anos & 5,2 & \pm & 1,6 & 4,5 & 5,8 & 9,6 & \pm & 3,9 & 8,1 & 11,2 \\
\hline 50 anos ou mais & 4,5 & \pm & 0,8 & 4,0 & 5,0 & 9,6 & \pm & 3,6 & 7,1 & 12,1 \\
\hline \multicolumn{11}{|l|}{ Escolaridade } \\
\hline Ensino fundamental (in)completo & 4,7 & \pm & 0,9 & 4,2 & 5,2 & 10,1 & \pm & 3,2 & 8,3 & 11,9 \\
\hline Ensino médio (in)completo & 5,3 & \pm & 2,1 & 4,6 & 6,1 & 9,5 & \pm & 3,9 & 8,2 & 10,9 \\
\hline Ensino superior (in)completo & 4,7 & \pm & 1,3 & 3,9 & 5,5 & 10,6 & \pm & 3,8 & 8,3 & 12,9 \\
\hline
\end{tabular}

\section{Discussão}

Este estudo é um dos poucos que procura avaliar e comparar a violência pelo parceiro íntimo em abstinência e no período em que fazia abuso de álcool.

A presente pesquisa identificou que $64,0 \%$ das companheiras de homens que estavam em período de abstinência, deixaram de sofrer violência pelo parceiro. Neste estudo os dados encontrados não demonstraram relação significativa entre idade e violência. Tal achado pode ser explicado pelo fato de terem sido excluídos tanto homens como mulheres menores de 18 anos.

Talvez o fato da pesquisa ter sido realizada num centro de referência para álcool tenha certa influência sobre o resultado. Os casos mais simples em geral são manejados em Unidades Básicas de Saúde por médicos de família juntamente com sua equipe e dessa forma não chegam até o CAPSad.

Alguns resultados podem ter sido influenciados por fatores como o baixo número absoluto de sujeitos com nível superior (seja completo ou incompleto) e também pelo fato da cidade onde foi realizada a pesquisa apresentar um IDH (Índice de Desenvolvimento Humano) de 0,808 no ano de 2010 segundo o Instituto Brasileiro de Geografia e Estatística (IBGE), sendo um dos melhores índices do Brasil. Além disso, culturalmente na região estudada, sabemos que o número de pessoas de alta renda que procuram o sistema público de saúde é baixo (apesar de não haver dados da Secretaria de Saúde sobre a escolaridade dos pacientes atendidos).

As percepções que o paciente apresenta sobre o álcool (sociais e culturais) também podem desempenhar um papel no qual a aceitação e tolerância do mau comportamento relacionado ao álcool (inclusive a agressão) podem influenciar as expectativas sobre seu comportamento enquanto bebem. Ou seja, uma parte dos pacientes etilistas podem intencionalmente envolver-se em agressão ou violência porque têm a expectativa de que tal comportamento possa ser justificado com base no fato de que estavam bebendo no momento. ${ }^{5}$

A relação entre o nível de escolaridade e a violência praticada pelo parceiro íntimo e a violência sexual é complexa. A baixa escolaridade é fator consistente relacionado com a perpetração desse tipo de violência. ${ }^{15}$ Mulheres com baixo nível de instrução (fundamental ou nenhum) têm de 2 a 5 vezes mais risco 
de sofrerem violência praticada pelo parceiro íntimo que mulheres com alto nível de escolaridade. ${ }^{16}$ No entanto, outra pesquisa identificou que as mulheres com um alto nível de instrução também estavam em grande risco de sofrer violência praticada pelo parceiro íntimo. ${ }^{17} \mathrm{Ou}$ seja, existem estudos que demonstram relação da violência por parceiro íntimo com baixa escolaridade, enquanto outros apontam associação com a alta escolaridade. No presente estudo não se encontrou relação significativa entre o nível de escolaridade e violência. Assim, mais estudos são necessários sobre como o nível de escolaridade está associado a vários tipos de violência praticada pelo parceiro íntimo.

Em relação à idade, a juventude foi identificada como fator de risco para homens cometerem violência contra parceiras e para mulheres sofrerem violência praticada por parceiro íntimo. ${ }^{15} \mathrm{De}$ acordo com dados de sistemas da justiça e centros para crises por estupros no Chile, Malásia, México, Papua Nova Guiné, Peru e Estados Unidos, entre um a dois terços de todas as vítimas de agressão têm até 15 anos de idade. No entanto, tais dados podem ser tendenciosos visto que algumas formas de violência são estreitamente associadas às idades mais jovens, em particular a violência que ocorre nas escolas e colégios e o tráfico de mulheres para exploração sexual. Já para a perpetração de violência pelos homens, é menos claro que a juventude seja um fator de risco. Na África do Sul, foi sugerido que homens na faixa etária de 20-40 anos eram mais propensos a terem cometido estupro que outros homens mais jovens ou mais velhos. ${ }^{18}$

Variações importantes nos resultados podem ser explicadas por perfil sociodemográfico diferente. O fato da idade não apresentar relação significativa com os índices de violência vai contra a maioria das pesquisas, talvez pela população estudada ter características culturais específicas. A escolaridade também não demonstrou relação direta com a violência causada pelo parceiro íntimo, pois é possível que cada vez mais pessoas com maior grau de instrução começam a procurar ajuda e relatar episódios de violência.

Em relação às mulheres entrevistadas livremente, suas respostas corroboram o encontrado na pesquisa com o uso do questionário HITS, que demonstrou diminuição da violência pelo parceiro na maioria dos casos.

\section{Conclusão}

Este estudo mostrou que a violência por parceiro íntimo diminuiu na abstinência. Mesmo para aquelas mulheres com menos do que 10 pontos no questionário HITS (não caracterizando violência) houve decréscimo na pontuação. Apenas 15\% das entrevistadas relataram não haver diferença com o parceiro em abuso de álcool ou em abstinência.

A escolaridade e a idade das entrevistadas não demonstraram ter relação significativa com o nível de violência por parceiro íntimo.

Os achados deste estudo apontam para novas estratégias envolvendo a abstinência alcoólica e a violência praticada pelo parceiro. Novos estudos são necessários para esclarecer se realmente o fato do homem estar em abstinência é fator suficiente para diminuir a violência ou se mais aspectos estão envolvidos na questão.

\section{Contribuição dos autores}

Concepção e/ou delineamento do estudo: JKY,MDBC; Aquisição, análise ou interpretação dos dados: JKY,MDBC,LN,PI; Redação preliminar: JKY,MDBC; Revisão crítica da versão preliminar: JKY,MDBC,SMP. 
Todos os autores aprovaram a versão final e concordaram com prestar contas sobre todos os aspectos do trabalho.

\section{Agradecimentos}

Aos funcionários do CAPS de Maringá, em especial Leandro. À professora Rosangela Ziggiotti do departamento de Medicina.

\section{Conflito de interesses}

Declaram não haver.

\section{Referências}

1. Netto L de A, Moura MAV, Queiroz ABA, Tyrrell MAR, Bravo M del M. Violência contra a mulher e suas consequências. Acta Paul Enferm [Internet]. 2014;27(5):458-64. Available from: http://dx.doi.org/10.1590/1982-

2. Vieira LB, Cortes LF, Padoin SM de M, Souza IE de O, Paula CC de, Terra MG. Abuso de álcool e drogas e violência contra as mulheres: denúncias de vividos. Rev Bras Enferm. 2014;67(3):366-72.

3. Smith PH, Homish GG, Leonard KE, Cornelius JR. Intimate partner violence and specific substance use disorders: Findings from the National Epidemiologic Survey on Alcohol and Related Conditions. Psychol Addict Behav. 2012;26(2):236-45. DOI: https://doi.org/10.1037/ a0024855

4. Cunradi CB, Mair C, Todd M. Alcohol Outlet Density, Drinking Contexts and Intimate Partner Violence: A Review of Environmental Risk Factors. J Drug Educ. 2014;44(0):19-33. DOI: https://doi.org/10.1177/0047237915573527

5. Wilson IM, Graham K, Taft A. Alcohol interventions, alcohol policy and intimate partner violence: a systematic review [Internet]. 2014. Available from: http://www.biomedcentral.com/1471-2458/14/881 DOI: https://doi.org/10.1186/1471-2458-14-881

6. Afifi TO, Henriksen CA, Asmundson GJG, Jitender S. Childhood Maltreatment and Substance Use Disorders Among Men and Women in a Nationally Representative Sample [Internet].Vol. 57, The Canadian Journal of Psychiatry. 2012. Available from: www.TheCJP.ca DOI: https://doi.org/10.1177/070674371205701105

7. Chamorro J, Bernardi S, Potenza MN, Grant JE, Marsh R, Wang S, et al. Impulsivity in the general population: A national study. J Psychiatr Res. 2012;46(8):994-1001. DOI: https://doi.org/10.1016/j.jpsychires.2012.04.023

8. Silva ACLG, Coelho EBS, Moretti-Pires RO. O que se sabe sobre o homem autor de violência contra a parceira íntima: uma revisão sistemática. Vol. 35, Rev Panam Salud Publica. 2014.

9. Miot HA. Tamanho da amostra em estudos clínicos e experimentais. JVsac Bras. 2011;10(4):275-8. DOI: https://doi.org/10.1590/S167754492011000400001

10. Sherin KM, Sinacore JM, Li XQ, Zitter RE, Shakil A. Hits: A short domestic violence screening tool for use in a family practice setting. Fam Med. 1998 Jul;30(7):508-12.

11. Iverson KM, King MW, Gerber MR, Resick PA, Kimerling R, Street AE, et al. Accuracy of an Intimate Partner Violence Screening Tool for Female VHA Patients: A Replication and Extension. J Trauma Stress [Internet]. 2015 Feb;28(1):79-82. Available from: http://doi.wiley. com/10.1002/jts.21985 DOI: https://doi.org/10.1002/jts.21985

12. Singh V, Petersen K, Singh SR. Intimate partner violence victimization Identification and response in primary care. Vol. 41, Primary Care - Clinics in Office Practice. W.B. Saunders; 2014. p. 261-81. DOI: https://doi.org/10.1016/j.pop.2014.02.005

13. Chen PH, Jacobs A, Rovi SL. Intimate partner violence: office screening for victims and perpetrators of IPV. FP Essent. 2013;412:11-7. PMID: 24053260

14. Fonsêca L de MA. Tradução, adaptação cultural e propriedades psicométricas da hurt insult threatened scream para rastreio da violência doméstica contra idosos no Brasil [Internet]. Santa Cruz; 2018. Available from: https://repositorio.ufrn.br/jspui/bitstream/123456789/25211/1/ LuizaDeMarilacAlvesDaFonseca_DISSERT.pdf 
15. Ally EZ, Laranjeira R, Viana MC, Pinsky I, Caetano R, Mitsuhiro S, et al. Intimate partner violence trends in Brazil: data from two waves of the Brazilian National Alcohol and Drugs Survey. Rev Bras Psiquiatr. 2016;38:98-105. DOI: https://doi.org/10.1590/1516-4446-20151798

16. WHO I Global status report on violence prevention 2014. World Health Organization. Geneva: World Health Organization, United Nation Development Programme, United Nations Office on Drugs and Crime; 2014.

17. Flake DF. Individual, family, and community risk markers for domestic violence in Peru. Violence Against Women [Internet]. 2005 Mar;11(3):353-73. Available from: http://www.ncbi.nlm.nih.gov/pubmed/16043554 DOI: https://doi.org/10.1177/1077801204272129

18. Jewkes R, Sikweyiya Y, Morrell R, Dunkle K. Understanding men's health and use of violence: interface of rape and HIV in south Africa. MRC Policy Brief. Pretoria; 2009. 